\title{
Téoros
}

Revue de recherche en tourisme

\section{Le pèlerinage}

Aux limites du religieux et du tourisme

\section{Mathieu Boisvert}

Volume 16, numéro 2, été 1997

Tourisme et religion

URI : https://id.erudit.org/iderudit/1074572ar

DOI : https://doi.org/10.7202/1074572ar

Aller au sommaire du numéro

Éditeur(s)

Université du Québec à Montréal

ISSN

0712-8657 (imprimé)

1923-2705 (numérique)

Découvrir la revue

\section{Citer cet article}

Boisvert, M. (1997). Le pèlerinage : aux limites du religieux et du tourisme.

Téoros, 16(2), 5-9. https://doi.org/10.7202/1074572ar

Ce document est protégé par la loi sur le droit d'auteur. L'utilisation des services d'Érudit (y compris la reproduction) est assujettie à sa politique d'utilisation que vous pouvez consulter en ligne.

https://apropos.erudit.org/fr/usagers/politique-dutilisation/
Cet article est diffusé et préservé par Érudit.

Érudit est un consortium interuniversitaire sans but lucratif composé de l'Université de Montréal, l'Université Laval et l'Université du Québec à Montréal. Il a pour mission la promotion et la valorisation de la recherche. https://www.erudit.org/fr/ 


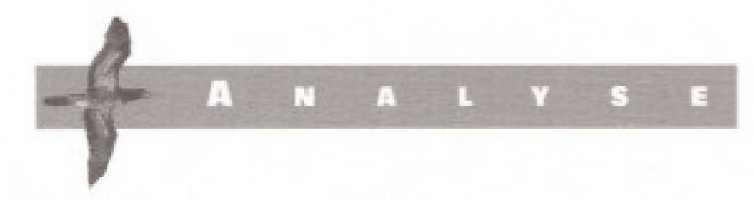

\section{Le PèLerinage}

\section{AUX LIMITES DU RELIGIEUX ET DU TOURISME ${ }^{1}$}

\section{Mathieu Boisvert, professeur Département des sciences religieuses - UQAM}

* C'est un pèlerinage? " demanda-t-il. Je dis que non. Ou plutôt que si, mais pas au sens religieux du terme. C'était tout au plus un itinéraire privé, comment dire ? Je cherchais seulement des traces. [...]

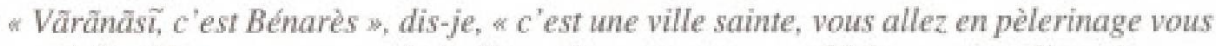
aussi ? Mon compagnon éteignit sa cigarette et toussa legerrement. * J'y vais pour mourir s (Tabucchi, 1987: 47-48).

Depuis quelques mois déjà, un groupe de seize étudiants de premier cycle de l'UQAM met en place les structures qui leur permettront d'effectuer une session d'étude en Inde sous la thématique du pèlerinage. Les pèlerins du savoir, comme ils aiment se nommer, visiteront plusieurs sites de pelerinage, tant bouddhistes, hindous et jains que musulmans. Avant qu'ils ne s'engagent dans ce périple, cependant, ils suivront deux cours sous la responsabilité d'une équipe de quinze professeurs d'universités québécoises puis, une fois en Inde, deux autres cours donnés par des intervenants universitaires indiens. Puisque ce projet exige un déplacement à l'étranger, il possède en soi les trois caractéristiques essentielles du pélerinage, soit le retrait du contexte habituel, l'expérience en lieu de pèlerinage et la réagrégation ${ }^{2}$. Nous pourrions même avancer que ce voyage d'étude est lui-même un pèlerinage.

Cependant, une question demeure : est-ce bien un pèlerinage, ou simplement une activité touristico-académique ? Le pèlerinage est-il une activité qui s'inscrit nécessairement au sein d'une tradition religieuse ou s'insère-t-il dans une démarche séculière ? Un nord-américain qui s'engage dans un voyage touristique des sites sacrés du sous-continent indien est-il un pèlerin? Et le pèlerin musulman en route vers la Mecque n'est-il pas également un touriste ? Cet article tentera done de cemer la circonférence délimitant chacune des sphères d'activité que sont le pèlerinage et le tourisme. Pour sonder plus à fond cette problématique, nous proposons d'abord l'analyse des trois étapes successives qui semblent inhérentes à tout pèlerinage. Nous appliquerons ensuite chaque étape à la session d'étude en Inde, au pèlerinage religieux, ainsi qu'au tourisme. Cette démarche nous permettra, tout au moins, de clarifier la distinction, si subtile soit-elle, entre le pèlerin et le touriste.

Le pèlerinage nécessite une séparation de notre contexte habituel, une distanciation temporaire qui ne peut se faire sans mouvance. En effet, le déplacement est la première image qui vient à l'esprit lorsque l'on songe au pèlerinage ; que ce soit en
Europe médiévale où pèlerin signifiait étranger, woyageur (pelegrinus), ou en Inde où le pèlerin est celui qui va (yätrika), la mouvance constitue l"activité principale du pèlerin, une dérmarche sur laquelle plusieurs traditions monastiques ont fondé leurs pratiques. Reportonsnous aux stades de la vie chez les hindous qui structurent l'existence de chaque individu en quatre périodes précises, dont la dernière est un renoncement total symbolisé par une itinérance constante, d'où le nom de parivrajaka [pari = autour ; $v r a j=$ aller] attribué aux premiers ascètes indiens. Le mot anachorète (anachörein), pour sa part, que l'on utilisait à l'origine pour désigner les fraudeurs fiscaux qui fuyaient les villes de peur d'être retracés par le fisc romain en est venu, au fils du temps, à désigner les premiers ermites chrétiens du quatrième siècle. Pensons également aux premiers moines irlandais qui, régis par le concept de xênitêia, se dếplaçaient constamment afin de développer le sentiment d'être étrangers à ce monde, de simples passants exilés de leur terre d'origine.

En examinant l'histoire, nous découvrons que la majorité des traditions religieuses perçoivent la pérégrination comme une pratique importante. En effet, la rupture est généralement considérée comme l'un des éléments responsables de l'émergence d'une nouvelle perspective, la mise en branle d'une transformation interieure (Monod, 1936 ; 385-399 ; Roussel, 1954). Le pèlerin n' 6 chappe point à cette dynamique : il s'approprie, réactualise temporairement cet idéal monastique en se sevrant temporairement de son entourage habituel ; il rompt les liens avec sa communauté immédiate afin d'aller vers. 


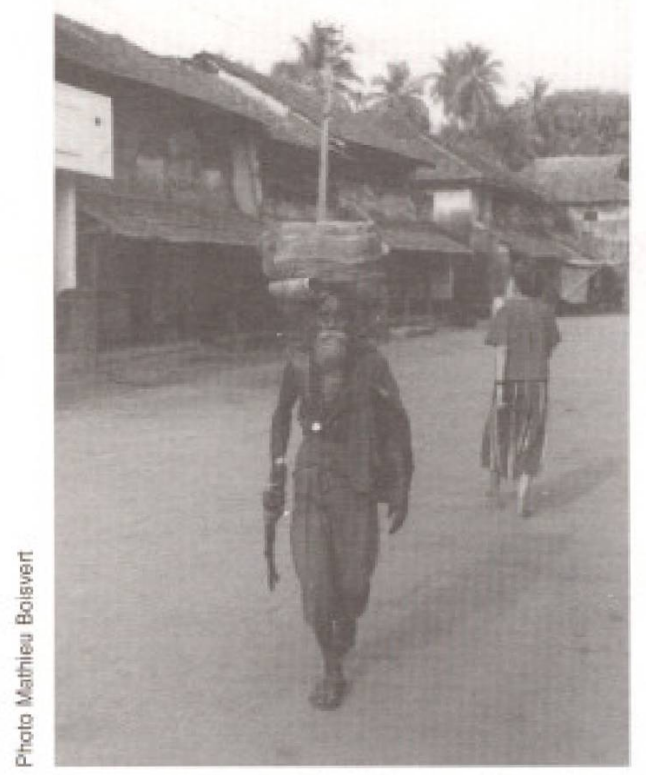

Rencontre d'un ascète et d'une occidentale...

Le pèlerin se sépare, se retire provisoirement de son contexte familial, social, économique et de ses activités quotidiennes afin d'entreprendre une démarche individuelle. Celle-ci devient également collective lors du stade liminal, car partagée par l'ensemble des individus qui participent au périple.

Dans l'exégèse d'un passage de la Genèse ( $\propto$ Quitte ton pays, ta famille, la maison de ton père, pour le pays que je te montrerai $»[\mathrm{Gen} .12 .11)$, Jean Cassien souligne trois types de renonciation que les monastiques doivent accomplir : l'entretien d'une abnégation complète des biens matériels, symbolisée par « quitte le pays $\$$; la renonciation de vieilles habitudes que nous cultivons depuis la naissance, représentée par \& la famille *; puis un rejet de tout souvenir lié à la communauté que le moine ou la moniale a quittée, symbolisé par \& la maison de ton père $»$. Le pèlerin incarne donc, momentanément, un des idéaux monastiques, car il se dépayse en laissant derrière lui l'univers de sens dans lequel il baignait. En cette aube de troisième millénaire, pensons au musulman new-yorkais cheminant vers la Mecque, ou au juif marocain en route vers Jérusalem, tous deux régis par des règles différentes de celles qui gouvernaient leur quotidien : ils doivent ểre vêtus d'une façon particulière, accomplir certains rituels, s'abstenir de certaines actions, etc. Cependant, et d'une perspective purement phénoméno- logique, la rupture en elle-même est effective puisqu'elle permet au pèlerin, de par la distance qu'elle crée entre lui et ses activités régulières, de jeter un regard nouveau sur son existence.

Cette rupture est nécessaire non seulement pour permettre une distanciation par rapport aux activités quotidiennes, mais également pour enclencher le cheminement vers un centre de pèlerinage, un axis mundis, un lieu façonné par plusieurs siècles d'histoire, véhiculant ainsi une dimension privilégiée pour le pèlerin. En d'autres termes, cette rupture engendre l'établissement de liens avec une dimension plus large, une dimension qui n'est plus régie par le milieu immédiat, mais plutôt par un contexté quasi mythique, en dehors du temps et de l'espace. Le pèlerin se soustrait de ses activités quotidiennes pour participer à un autre type de pratiques déterminées, cette fois, non pas par la quotidienneté et l'individualitê, mais par un enracinement au cœur d'une tradition et partagées par l'ensemble d'une communauté. Par exemple, plus de trois millions de pèlerins cheminent vers la Mecque chaque année. Ceux-ci proviennent de contextes culturels très différents ; certains habitent l'Amérique, d'autres l'Asie, l'Afrique ou l'Europe. Issus de milieux distincts, ils partagent cependant un profond univers de sens : la Mecque représente l'endroit où aurait débuté la création, l'emplacement où $\mathrm{Ibrhm}$ (Abraham) aurait construit la première maison de dévotion (la $\left.K a^{\prime} b a h\right)$, et le lieu que tout musulman se doit de visiter une fois dans sa vie ${ }^{3}$. Comme l'affirme le Coran,

Souviens-toi que nous avons assigné à Abraham l'emplacement de la maison sainte, en lui disant : Ne nous associe aucun autre Dieu dans ton adoration ; conserve cette maison pure pour ceux qui viendront $y$ faire des tours de dévotion, qui s'y acquitteront des œuvres de piété debout, agenouillés ou prosternés.

Annonce aux peuples le pélerinage [sic] de la maison sainte, qu'ils y arrivent à pied ou montés sur des chameaux prompts à la course, venant des contrées eloignées [XXII, 27-28] (Le Coran, 1980 : 267).

La tradition bouddhiste affirme également que certains lieux doivent être visi- tés par ceux qui désirent approfondir l'enseignement du Bouddha. Ainsi, l'endroit où Siddhartha Gautama serait né, le lieu où il aurait atteint l'illumination, l'emplacement où il aurait promulgué son premier discours et la ville où il serait décédé sont tous des endroits clés que les pieds des dévots, provenant de tout les continents, doivent fouler (DTghaniākya, II ; 140). Ainsi, la majorité des grandes traditions religieuses établissent, au cours des siècles, des sites de pouvoir que le dévot est encouragé à visiter. Pensons entre autres à Jérusalem, Rome et Compostelle, pour le christianisme, et aे Vãranãsĩ, Mathurä-Vndãvana ou Kã̃i cipuram, pour l'hindouisme. La visite dévotionnelle de ces sites permet au pèlerin d'actualiser la tradition, de situer sa propre existence dans une longue, très longue continuité historique.

Bien que le pèlerinage exige une rupture, un déplacement, il rend également possible la création d'une nouvelle communauté à l'intérieur de laquelle un nouvel univers de sens est partagé, une communauté trans-historique, car ses fondements sont souvent enracinés dans le mythe ou l'hagiographie. Les bouddhistes qui se dirigent vers Lumbini pour rendre hommage au lieu de naissance du Bouddha réactivent et participent à un imaginaire qui, généralement, demeure latent. Dans son autobiographie, Malcom $\mathrm{X}$ remarque cette communauté, diversifiéc de par les provenances culturelles, mais unifiée par sa croyance :

Jamais (écrivis-je) je n'ai connu d'hospitalité aussi sincère, de fraternité aussi bouleversante que celles des hommes et des femmes de toutes races réunis sur cette vieille terre sainte, patrie d'Abraham, de Mahomet et des autres prophètes des Saintes Écritures. Jamais je n'ai été honoré comme ici. Jamais je ne me suis senti plus humble et plus indigne. L'Amérique a besoin de comprendre l'Islam, parce que c'est la seule religion qui ignore le racisme. Ce pèlerinage m'a obligé à réviser certaines idées qui étaient miennes, à rejeter certaines conclusions auxquelles j'étais parvenu. Au cours des onze journées que j'ai passées ici, dans le monde musulman, j'ai mangé dans le même plat, bu dans le même verre, dormi dans le même lit (ou sur le même tapis), j'ai prié le même Dieu - que mes coreligion- 
naires aux yeux les plus bleus, aux cheveux les plus blonds, à la peau la plus blanche. Dans leurs paroles comme dans leurs actes, les Musulmans a blancs * sont aussi sincères que les Musulmans \& noirs d'Afrique - nigériens, soudanais, ghanéens (Malcom X, 1993 ; 280-281).

Bien que cette nouvelle communauté de pèlerins soit éclectique et disparate, un lien étroit unit les membres. Ils actualisent ensemble une tradition, ils prennent conscience d'un fil conducteur qui part du présent et remonte à un temps immémorial. Ainsi, par la rupture inhérente à l'activité du pèlerinage, le pèlerin tisse de nouveaux liens qui lui permettent de remplacer ceux qui furent temporairement sevrés, mais, plus important encore, de percevoir le lieu de pèlerinage comme sacré, constituant ainsi un seuil spatial privilégié. Tel que le souligne Victor Turner,

Un limen est, bien sûr, au sens littéral un a seuil $\%$. Un centre de pèlerinage, du point de vue de l'acteur croyant, représente aussi un seuil, un lieu et un moment a dans le temps et hors du temps $\mathrm{x}$, et cet acteur - comme l'attestent de nombreux pèlerins de nombreuses religions - espère y avoir une expérience directe du sacré, invisible, ou de l'ordre surnaturel, soit sous l'aspect matériel d'une guérison miraculeuse, soit sous l'aspect immatériel d'une transformation intérieure de l'esprit ou de la personnalitét.

Que des milliers d'individus se déplacent vers un même centre, lui attribuent tous une même signification, partagent tous un objectif commun, fait en sorte que cet endroit incarne une dimension particulière qui peut stimuler d'intenses expériences.

Quand l'objectif est atteint, le lieu de pèlerinage, le pèlerin quitte l'espace liminal et rompt à nouveau les liens, mais cette fois avec la communauté pèlerine, afin de réintégrer son environnement social de départ. La transformation de l'individu prend place au moment de la réagrégation, lorsqu'il revient dans sa communauté locale. Tout pèlerinage mène à une transformation ou, tout au moins, à la confirmation d'un univers de sens dont les fondements s'enracinent dans une lointaine tradition".
A la suite de cette description succincte du pèlerinage, nous est-il possible de situer le tourisme par rapport à cette activité ? Tout comme le pelerin, le touriste se retranche temporairement de sal communauté, que ce soit pour écourter le pénible hiver canadien en séjournant dans les tropiques ou pour visiter SaintPierre de Rome à la veille de l'année jubilaire 2000. Dans leur pérégrination, le pèlerin et le touriste créent tout deux une distance par rapport à leur milieu d'origine, un recul qui justement permet de mettre en perspective leur existence respective. Cependant, et de manière générale, le tourisme ne permet pas la création de liens aussi profonds et soutenus entre les participants d'un même voyage. Puisque ceux-ci ne partagent pas d'objectif commun, régi par un code spécifique et pratiquement transhistorique, les liens développés n'ont pas la même portée. Un groupe de pèlerins hindous qui, remontant le Gange jusqu'à sa source, est conscient que ce périple a été effectué à maintes reprises depuis des siècles par des individus partageant de semblables préoccupations religieuses ne peut que conférer une dimension particulière au voyage. En visitant le lieu de naissance de leur prophète, Joseph Smith, un groupe de jeunes mormons de l'Utah ne peut en ressortir qu'avec une foi renouvelée et une conscience fortifiée de communauté. Cependant, un groupe d'ornithologues qui prépare depuis plusieurs mois une expédition dans les forêts vierges de Costa Rica pour y étudier de rares espèces de volatiles peut très facilement $s^{*}$ apparenter à un pèlerinage : une communauté d'individus partageant les mêmes préoccupations (dans ce casci. les oiseaux) s'engage dans un périple avec un objectif précis : recueillir plus d'information sur de rares espèces. Le voyage se prépare à l'avance et culmine en un point précis de la jungle équatoriale. En outre, il transporte le paraphe propre aux ornithologues, les distinguant ainsi des vulgaires touristes, tout comme la coquille Saint-Jacques démarque le pèlerin du simple visiteur. Le retour des ornithologues peut également ếtre marqué par une transformation. Cependant, les liens qui les unissent ne sont pas enracinés dans une lointaine tradition. Bien sûr, l'ornithologie est une science et, en ce sens, également une tradition ; néanmoins, cette tradition se préoccupe des oiseaux plutôt que des aspirations et des craintes de ses adhérents. Elle ne pos- sède aucune théodicée, en ce sens qu'elle n'offre aucune solution aux profondes questions existentielles qui peuvent parfois surgir ; l'ornithologie ne véhicule aucun concept similaire à ceux de karma ou de dieu bienveillant. La distinction principale entre un pèlerinage et une exploration ornithologique, selon nous, est le type de liens entre les différents participants. Dans le cas du pèlerinage, ces liens doivent d'abord avoir une importante ampleur historique ou avoir contribué à structurer les fondements d'une communauté précise (pensons à l'incidence du catholicisme au Québec, du bouddhisme en Thaillande, du confucianisme en Chine, ou encore de l'islam en Iran). Ces mêmes liens doivent également avoir une dimension sotériologique, un objectif de transformation, voire mểme de libération, aussi partielle soitelle.

Les pèlerins du savoir, auxquels nous faisions allusion au debut de cet article, se rapprochent probablement plus des pèlerins traditionnels que des ornithologues. Tout comme ces derniers, ils se distancieront sous peu de leur communauté immédiate afin de s"engager dans un voyage. En élaborant une campagne de financement et en assimilant de l'information sur la culture indienne, l'hindouisme, le bouddhisme et autres religions que l'on trouve en Inde, ils fortifient également les liens qui les unissent mutuellement. Il importe de noter que ces liens ne sont pas seulement intellectuels, mais également émotifs, puisque les principes de gestion participative sont au centre du projet d'étude et que les participants sont contraints à des confrontations individuelles et collectives. Pendant leur séjour en Inde, cependant, ces liens ne pourront éliminer la distance qui les sépare de l'ascète hindou cheminant vers la Kumbha mela ${ }^{6}$.

Les pèlerins du savoir ne sont pas indiens et ne prétendent pas participer entièrement à l'univers de sens qui meut d'innombrables masses sur le sous-continent sud-asiatique. Cependant, au sein du groupe, d'importants liens se tisseront autour d'un objectif commun : se familiariser avec la mosaïque religieuse indienne et percevoir les incidences sociales et culturelles de cette dernière sur la société. Bien que ces liens ne soient pas issus d'une réalité trans-historique, mythique ou hagiographique, pourrait-on 
affirmer qu'ils s'inscrivent tout de même dans une mythologie du savoir, dans une quête pérenne d'identité et de localisation de celle-ci dans le temps et l'espace ?

Un autre élément pourrait peut-être nous aider à cemer la circonférence de ces activités. Le pèlerinage est une activité, principalement laiqque, au sein de laquelle se trouvent certaines caractéristiques du monachisme, tant occidental qu'oriental. Tout comme le moine, le pélerin doit généralement prononcer des voux. Ceuxci varient d'une tradition à l'autre, mais nous pouvons affirmer que le célibat est un dénominateur commun. Le pèlerin bouddhiste, hindou, musulman ou chrétien se doit d'observer cette règle, non parce que la sexualité est perçue comme mauvaise, mais plutôt, comme le disait saint Paul, parce que celle-ci met en relation intense des individus at que les responsabilités mutuelles ne peuvent être évacuées :

Elle passe, en effet, la figure de ce monde. Or, je vous voudrais exempts de soucis. L'homme non marié a souci des choses du Seigneur, des moyens de plaire au Seigneur. Mais celui qui est marié a souci des choses de ce monde, des moyens de plaire à sa femme, et il est partagé. De même, la femme non mariée, comme aussi la jeune fille, a souci des choses du Seigneur pour être sainte de corps et d'esprit ; mais celle qui est mariée a souci des choses de ce monde, des moyens de plaire à son mari. Je dis cela dans votre propre intérêt, non pour vous tendre un piège, mais (pour vous porter) vers ce qui est bienséant et de nature à vous attacher au Seigneur sans partage ( 1 Cor. $7: 31$ 35 ).

Le pèlerin prend donc ses voux pour mieux se concentrer sur son objectif principal, afin de devenir, comme les monastiques de différentes traditions, monotrope, de ne plus avoir qu'une orientation, de ne penser qu'à une chose, que celle-ci soit l'atteinte du nirväna, la connaissance de Dieu, ou l'espoir de fouler la terre sainte. Bien sûr, les ornithologues doivent aussi épouser des préceptes précis sans quoi les oiseaux qu'ils pourchassent pourraient leur fausser compagnie. Les pèlerins du sawoir ont également endossé un contrat et élaboré des règles de comportement dans le but d'éviter de

\section{LES PÈLERINS DU SAVOIR}

Dans un contexte où l'intemationalisation des marchés devient de plus en plus présente et où une compréhension de la diversité culturelle est essentielle au fonctionnement social québécois, cette session d'étude en Inde permettra aux étudiantes et aux etudiants d'interagir avec une altérité sociale et culturelle, de développer les qualités requises pour une compréhension de cette différence, de mieux comprendre leur propre identité culturelle et de parfaire leur capacitế de fonctionner en équipe. A moyen terme, ces qualités contribueront, nous en sommes convaincus, à former des individus conscients de leur propre importance sociale ainsi que dotés d'outils pour mener à terme les différents objectifs (sociaux, professionnels ou autres) qu'ils se seront fixés.

L'Inde fut choisie comme contexte favorable à ce projet pour deux raisons principales. Bien que la culture indienne, tout comme celle de bien d'autres pays, soit radicalement différente de celle du Québec, le sous-continent sud-asiatique peut cependant être perçu comme un microcosme au sein duquel nous trouvons un amalgame culturel des plus diversifiés. D'autre part, par l'omniprésence du religieux dans la société indienne, ce pays constitue le contexte rêvé pour l'exploration de la présente thématique, le pèlerinage.

Les objectifs de ce projet seront poursuivis a travers la thématique du pelerinage, ce qui permettra de développer un contact étroit avec la culture indienne. Ainsi, les étudiantes et les étudiants seront amenés à résider en plusieurs endroits considérés cruciaux par les traditions religieuses indiennes et à discuter avec des professeurs d'universites indiennes, ainsi qu'avec les moines, les moniales et les ascètes visitant les mêmes sites de pèlerinage qu'eux.

La session INDE - hiver 1998 est une session complète de 15 crédits universitaires.
Elle commencera officiellement au tout début de janvier, où deux cours seront donnés de façon intensive (Hindouisme et Pelerinage- $I$ ) ; deux autres cours seront offerts en Inde durant les mois de février et mars (Bouddhisme et Pèlerinage-lI) ; les trois derniers crédits seront accordés pour la préparation du projet (dont la campagne de financement), pour l'apport au groupe, ainsi que pour les activités de retour à la collectivité, après le retour a Montréal. Cependant, les activités préparatoires au projet, entamées en février 1997, requièrent approximativement dix heures par semaine de collaboration de tous les participants.

Ce type de projet peut être perçu comme novateur dans le milieu académique québécois ; cependant, il importe de noter que la nouvelle association québécoise CEGEP international, ainsi que des universités américaines (State University of New York [S.U.N.Y. ] et Antioch University (Buddhist Studies in India Program I) ont déjà pressenti les avantages pédagogiques d'un tel projet.

Cette session d'étude s'adresse à toutes les étudiantes et tous les étudiants de premier cycle de I'UQAM. Le groupe est limité à 16 personnes afin de faciliter l'interaction, le contact avec la population indienne et la mobilité en Inde. Le groupe est constitué depuis le 7 avril et une sérieuse campagne de financement est déjà amorcée. Nous misons sur l'engagement étudiant pour que l'ensemble des frais soient recueillis. Les étudiants seront accompagnés d'un professeur ( $\mathrm{Ma}$ thieu Boisvert), d'un guide français expérimenté (Yves Bourgeons, Paris), et de deux responsables de groupe. M. Boisvert et $\mathrm{Y}$. Bourgeons seront tous deux responsables d'établir les liens préalables nécessaires avec les différents intervenants indiens pour le cours Pelerinage-II. compromettre les objectifs poursuivis. Bien que ni la tonsure, ni le célibat ne soient exiges de chacun de ces deux groupes, ils détiennent tout de même une codification qui leur est propre et qu'ils. doivent tous respecter afin d'atteindre leurs objectifs.

Moine temporaire, le pèlerin rompt les liens avec sa communauté, s'engage dans un périple, tisse de nouveaux liens, inter- prète son expérience en fonction de la nouvelle collectivité à laquelle il participe activement et revient transformé. Le simple touriste, quant à lui, rompt avec son environnement, mais ne crée pas nécessairement une nouvelle communauté dont les racines se perdent dans l'histoire. Les pelerins du savoir, eux, visiteront $d$ 'important sites religieux, mais ne parviendront pas à assimiler toute la signification que ceux-ci revêtent 


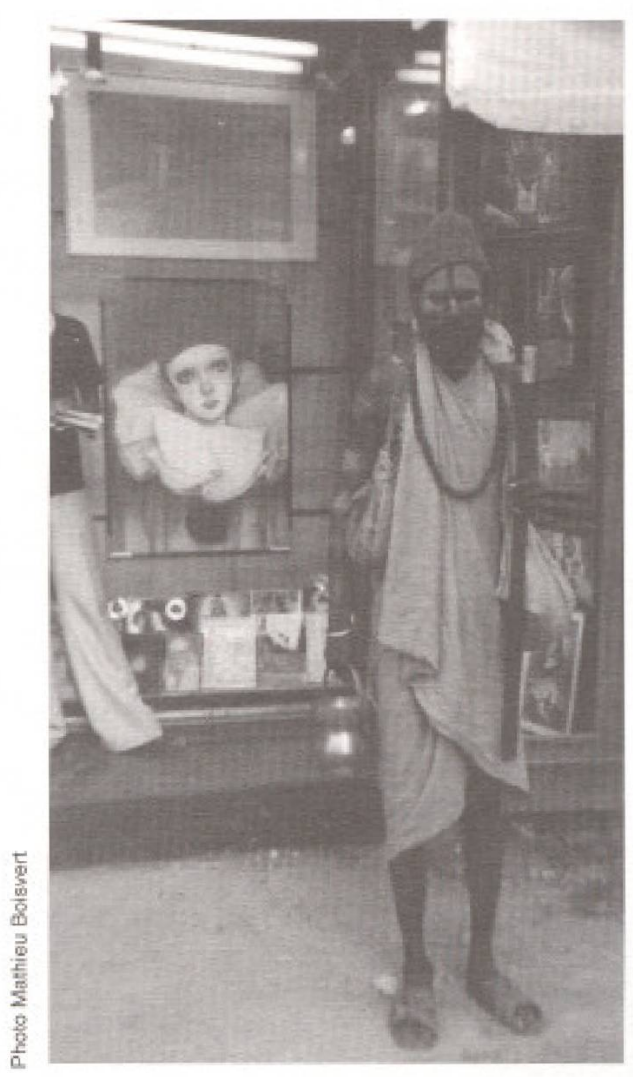

pour les indiens qui y séjournent religieusement. Cependant, en raison de la fascination occidentale envers l'Inde depuis déjà quelques décennies, ne pourrionsnous pas affirmer qu'un passé social et collectif, bien que relativement court, véhicule déjà un imaginaire mythique rattaché à l'Inde ? Pourrions-nous également y voir une tranche de notre histoire [beatniks, hippies, Beatles, révolution tranquille, nouvel âge] dans laquelle germent les fondements d'une redéfinition du pèlerinage et du tourisme ?

En guise de résumé, disons que le pèlerinage est une activité centralisatrice dont le noyau se situe à l'extérieur du quotidien et remonte à un lointain passé. Nous laissons cependant à d'autres le soin de déterminer les limites du quotidien et de ce lointain passé, nous assurant simplement de rappeler la maxime des alchimistes : « il n'y a de centre nulle part, que des circonférences... \%.

Un comité de lecture a lu et accepté ce texte

\section{NOTES}

1 Les idées présentées dans cet article furent générées, entre autre, par les vifs et stimulants échanges que j'ai eu l'opportunité d'avoir avec Monsieur Yves Racicot.

2 Selon la grille élaborểe par Victor Turner (1974, 167-230), et reprise par Edith Turner (327-330) et par David Carrasco (27-41).

3 Nous trouvons, dans la tradition islamique, cinq préceptes, cinq piliers qui doivent être respectés par tout musulman. Parmi ceuxci, se trouve le hajj, le pèlerinage à la Mecque.

4 Turner, $1974:$ 196-197. Traduction tirée de Carrasco, $1997: 29$.

5 Il importe de remarquer qu'une négation d'un univers de sens, résultant d'un pèlerinage ou de tout autre contexte, est également une affirmation, car elle porte en elle le positionnement d'un individu par rapport à une situation donnée. Pensons à la tradition dévotionnelle hindoue (bhakti) où quatre types de relation à la divinité sont permis : amant — amant, ami — ami, enfant parent, et celui fondé sur une relation de haine constante envers la divinité. L'objectif d"un bhakta est de n'avoir que la divinité en son esprit, peu importe le mode relation= nel privilégié.

6 La Kumbha melã est un pèlerinage hindou qui a lieu quatre fois tous les douze ans. chaque fois dans une des locations du nord de l'Inde : à Hardvãr, où le Gange sort des Himalayas, à Prayãg, où se rencontrent le Gange, la Yamun et l' $\alpha$ invisible Sarasvatì, à Ujiain, sur les bords de la rivière Kíprā, et à Nãsik, près de la rivière Godavari. La Kumbha mela est non seulement reconnue par la tradition hindoue comme étant un pelerinage où les karma peuvent être dissous et où d"incroyables mérites (puñia) peuvent ễtre accumulés. mais également comme un événement qui rassemble plusieurs communautés hindoues, permettant ainsi de débattre et de standardiser la doctrine et d'affirmer l'unité hindoue. D'après William S. Sax, la Kumbha melā est, démographiquement, le plus important pèlerinage du monde ; il arrive que plusieurs millions de pèlerins et d'ascètes hindous traversent quotidiennement le village où elle prend place (Sax, 401-402). Pour plus d'information sur les fondements des traditions monastiques orientales et occidentales, voir l'article de l'auteur \& A comparison of the Early Forms of Buddhist and Christian Monastic Traditions *, Boisvert, 1992: 123-143.

\section{BIBLIOGRAPHIE}

(1980), Le Coran, traduction et préface par Kasimirski, Paris, Editions Baudoin.

Boisvert, Mathieu (1992), \& A comparison of the Early Forms of Buddhist and Christian Monastic Traditions $\%$, Buddhist-Christian Studies, 12, 123-143.

Carrasco, David (1997), * Formes et diversité des pèlerinages w, Concilium, 266, p. 27-41.

Malcom X (1993), LAutobiographie de Malcom $X$, Paris, Bernard Grasset.

Monod, Victor (1936), * Les voyages, le déracinement de l'individu hors du milieu natal constituent-ils un des eléments déterminants de la conversion religieuse? * Revue d'histoire et de philosophie religieuse, 16, p. 385-399.

Roussel, R. (1954), Le pèlerinage à travers les siècles, Paris, Payot.

Sax, Willian S. (1987), * Kumbha mela $*$, Encyclopedia of Religion, New York, Macmillan and Free Press, 8, p. 401-402.

Tabucchi, Antonio (1987), Nocturne indien. Paris, Christian Bourgeois (6d.).

Turner, Edith (1987), * Pilgrimage : an overview 9. Encyclopedia of Religion, 11, p. $327-330$.

Turner, Victor (1974), Dramas, Fields, and Metaphors : Symbolic Action in Human Society, Ithaca, Cornell University Press, 